\title{
FORAMINÍFEROS TEXTULÁRIDOS EN SEDIMENTOS SOMEROS RECIENTES DE LA PENÍNSULA ANTÁRTICA Y SU RELACIÓN CON EL ÁREA SUBANTÁRTICA SUDAMERICANA ${ }^{1}$
}

\author{
TEXTULARIDS FORAMINIFERS FROM RECENT SHALLOW SEDIMENTS OF THE ANTARCTIC \\ PENINSULA AND THEIR RELATIONSHIP WITH SOUTHERN SOUTHAMERICAN REGION
}

\author{
Tatiana Hromic ${ }^{2}$, María Camblor² \& Lyta Quezada²
}

\begin{abstract}
Forty nine samples, of superficial marine sediments $(<50 \mathrm{~m}$.) from 3 localities: Deception Island, Chile Bay and Fildes Bay, Antarctic península were analysed in order to investigate the foraminiferers of suborder Textulariina Delage y Herouard, 1886. Twenty two species were identified and classiffied en 12 families and 15 generas. The most abundant species were Trochammina malovensis (53,2\%), Miliammina arenácea (16,3\%) y Reophax dentaliniformis (6,7\%). Deception Island contributed with most of the specimens (57\%) and Chile Bay with the highest number of species. Thes most common species in the area were Trochammina malovensis (95,9\% of the samples), Miliammina arenácea (73,5\%), Spiroplectammina biformis (63,3\%) and Trochammina squamata (61,2\%). Hierarchical cluster analysis and ANOSIM showed there is not communities differences between bays. Ten species (from 22) could not be found in the southernmost southamerica: Alveolophragmiun columbiense, Deuterammina glabra, Eggerella minuta, Pseudobolivina antarctica, Reophax nodulosus, Rhabdammina linnearis, Saccammina sphaerica, Textularia wiesneri, Trochammina malovensis and T. quadriloba. Probably they are endemic species for the Antarctic region.
\end{abstract}

Key words: Textulariina, shallow water, Deception Island, Fildes Bay, Chile Bay, Antártica.

1 Resultados parciales del Proyecto "Los Foraminíferos bentónicos antárticos y sus vinculaciones con la microfauna sudamericana" financiado por el Instituto Antártico Chileno.

2 Laboratorio de Micropaleontología, Instituto de la Patagonia, Universidad de Magallanes, Casilla 113-D, Punta Arenas, Chile. tatiana.hromic@umag.cl. 


\section{RESUMEN}

Se analizó un total de 49 muestras de sedimento marino superficial $(<50 \mathrm{~m})$ extraídas en tres localidades: isla Decepción, bahía Chile y bahía Fildes, península Antártica con el objeto de investigar los foraminíferos del suborden Textulariina, Delage y Herouard, 1886. Se identificaron 22 especies las que fueron clasificadas en 12 familias y 15 géneros. Las especies más abundantes fueron, Trochammina malovensis (53,2\%), Miliammina arenácea (16,3\%) y Reophax dentaliniformis (6,7\%). En isla Decepción se recolectó la mayoría de los ejemplares (57\%) y en bahía Chile, el número más alto de especies. La mayor distribución geográfica fue alcanzada por Trochammina malovensis (95,9\% de las muestras), Miliammina arenácea (73,5\%), Spiroplectammina biformis (63,3\%) y Trochammina squamata (61,2\%). El análisis de conglomerado y ANOSIM no mostraron diferencias significaticas de las comunidades de las bahías. De las 22 especies identificadas en las aguas antárticas ( $<50 \mathrm{~m}), 10$ no han sido citadas en la región austral del continente sudamericano: Alveolophragmiun columbiense, Deuterammina glabra, Eggerella minuta, Pseudobolivina antárctica, Reophax nodulosus, Rhabdammina linnearis, Saccammina sphaerica, Textularia wiesneri, Trochammina malovensis y T. quadriloba, por lo que podrían considerarse endémicas para la región antártica.

Palabras clave: Textulariina, sedimentos someros, Isla Decepción, bahía Fildes, bahía Chile, Antártica.

\section{INTRODUCCIÓN}

Entre los foraminíferos bentónicos más abundantes y diversos de la península antártica se encuentran los representantes del suborden Textulariina Delage \& Herouard, 1886 (Earland 1933, 1934; Heron Allen \& Earland 1932; Lena 1975, 1980; Finger \& Lipps 1981). Las especies de este taxa forman un caparazón con partículas de arena tomadas del medio, lo que les permite habitar en aguas con escasa disponibilidad de calcio y predominar en condiciones adversas para los foraminíferos calcáreos. Morfológicamente son muy variados, incluyendo formas mono o multicamerales, bi o triserial, con enrollamiento plani o trocoespiralado y con una o más aberturas (Loeblich \& Tappan 1988). La distribución y abundancia de poblaciones de estos organismos está afectada por el grado de estrés de los ambientes marinos (Murray 1991), en tanto que su productividad ha sido relacionada con el fotoperíodo de las estaciones de primavera y verano austral (Murray 2006).

Diversos componentes de las comunidades bentónicas marinas de las zonas australes han sido analizados para el estudio de las relaciones faunísticas entre Antártica y el cono sur de Sudamérica (e.g. Boschi \& Gavio 2005; Tatián et al. 2005; Zelaya 2005). En algunos casos, han surgido patrones biogeográficos relevantes (Véase Arntz et al. 2005, para una completa discusión al respecto). Sin embargo, el grupo de los foraminíferos no ha sido suficientemente analizado considerando, por ejemplo, distribuciones biogeográficas, no obstante la especificidad de las relaciones que este grupo establece con el ambiente (Kahio 1994; SenGupta \& Machain Castillo 1993, Smart 2002). A la fecha, se ha descrito una importante cantidad de especies de foraminíferos bentónicos para la región de Magallanes (e.g. Boltovskoy \& Totah 1987; Zapata \& Alarcón 1988; Violanti et al. 2000, Zapata \& Moyano 1997; Hromic (1996, 1997, 2001, 2002, 2011a, Hromic \& Zúñiga 2005; Zúñiga 2006) pero no se han establecido relaciones de esta fauna con la presente en los ambientes antárticos.

El objetivo de este estudio es describir la composición taxonómica y abundancia de los foraminíferos con caparazón arenáceo que habitan en 3-zonas someras $(<50 \mathrm{~m}$ ) en la península antártica (isla Decepción, bahía Chile y bahía Fildes), para determinar si hay diferencias entre las bahía y analizar sus vinculaciones y afinidades faunísticas con los foraminíferos australes reportados para la región de Magallanes.

\section{MATERIALES Y MÉTODOS}

Se recolectó un total de 49 muestras de sedimentos marino durante las expediciones ECA XXXII 


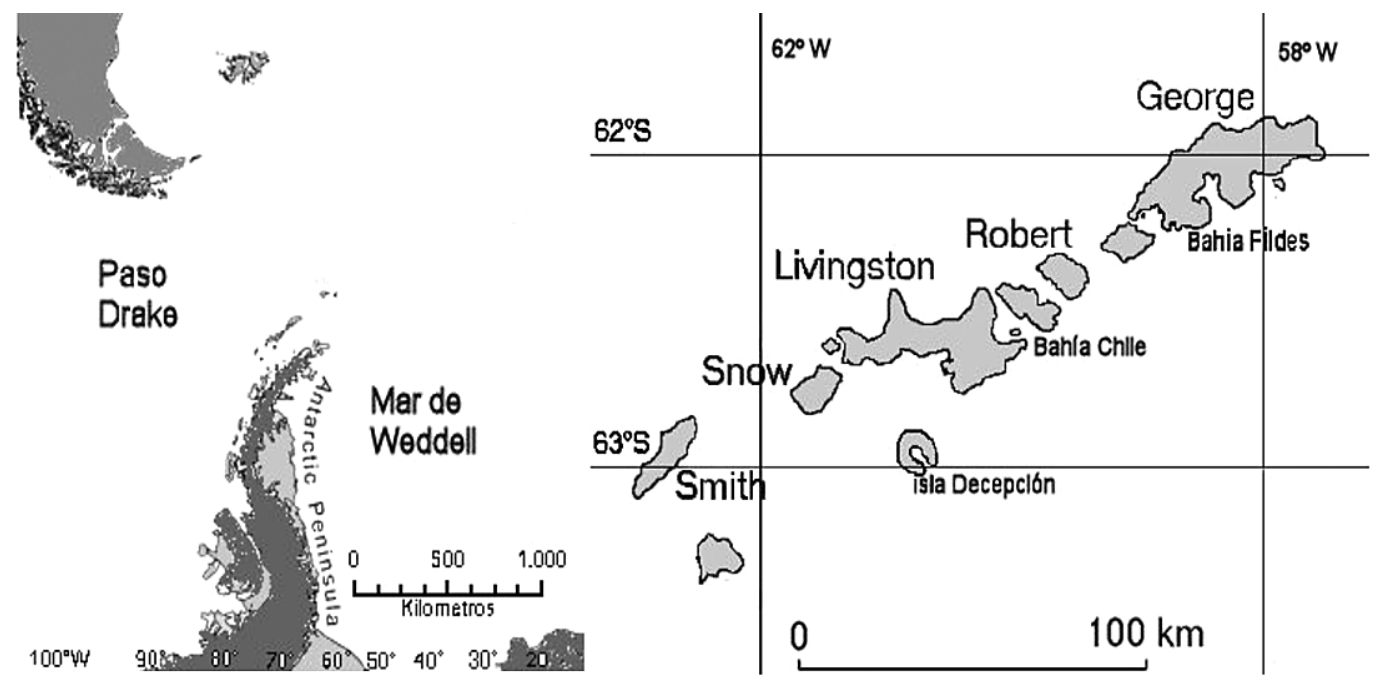

Fig. 1. Mapa general de las áreas de muestreo (para localización de los sitios de muestreo (ver Tabla 1).

(1996), ECA XXXIII (1997) y ECA XXXIV (1998), organizadas por el Instituto Antártico Chileno a la península Antártica en tres localidades; al interior de la bahía Fildes, bahía Chile e isla Decepción (Fig. 1; Tabla 1). El sedimento marino superficial fue extraído con draga Smith -McIntyre, desde profundidades entre 25 - $50 \mathrm{~m}$. De cada muestra, se separaron los 5 primeros centímetros para su posterior tratamiento. El material se lavó sobre un tamiz de malla de 63 micrones, se secó y luego se pesó 50 gramos para la extracción de la biota, siguiendo el procedimiento de Boltovskoy (1965). Los foraminíferos totales fueron extraídos uno a uno bajo lupa binocular, pegados en reglillas por forma y se identificaron siguiendo a Loeblich \& Tappan (1988) y Decrouez (1989) hasta nivel genérico. La determinación hasta nivel específico se basó en el catálogo de Ellis \& Messina (1940 et seq.) y en la literatura disponible para el área (Brady 1884; Barker 1960; Lena 1975, 1980; Boltovskoy et al. 1980; Heron- Allen \& Earland 1932; Earland 1933,
1934) o especializada en foraminíferos arenáceos (Höeglund 1947; Hermelin 1983). En forma paralela, los foraminíferos se contaron por especie.

El análisis de la información (matriz especies/ abundancia) se realizó utilizando el programa PRIMER 6.0 (Clarke \& Gorley, 2006). Para estimar la similitud/disimilitud de los foraminíferos recolectados en las tres localidades estudiadas (isla Decepción, bahía Chile y bahía Fildes), se realizó un análisis de conglomerados (Análisis de "Cluster") utilizando el índice de Bray-Curtis.

La significancia estadística de las agrupaciones obtenidas con el análisis de agrupamiento se evaluó con la rutina SIMPROF ("similarity profile") al 95\% de confianza. En este caso, la hipótesis nula evaluada es que el conjunto de muestras no difieren entre sí en términos de su estructura multivariada (conjuntos de foraminíferos bentónicos) y la hipótesis planteada es que si se encuenran diferencias significativas entre las comunidades faunística entre las bahías. A continuación se validó con MDS.

Tabla 1. Sitios específicos de muestreo en la Península Antártica, visitados en las tres expediciones ECA (¿?).

\begin{tabular}{lccc}
\hline Año & $\begin{array}{c}\text { Isla Decepción } \\
\text { Muestras }\end{array}$ & $\begin{array}{c}\text { Bahía Chile } \\
\text { Muestras }\end{array}$ & $\begin{array}{c}\text { Bahía Fildes } \\
\text { Muestras }\end{array}$ \\
\hline $\begin{array}{l}1996 \text { (ECA XXXII) } \\
\text { N } 16\end{array}$ & Dz; Db1; Dz1 y Db2 & Ch st1 & Fst1 y Fst2 \\
$\begin{array}{l}1997 \text { (ECA XXXII) } \\
\text { =20 }\end{array}$ & D1- D6 & Ch1- Ch8 & F1- F4 \\
1998 (ECA XXXIV) & De 1- De6 & Chi1- Chi8 & Fi 1- Fi 12* \\
\hline
\end{tabular}

*La muestra Fi5 no brindó foraminíferos 
Para estos análisis los datos de abundancia absoluta se transformaron a raíz cuarta para minimizar las diferencias de abundancia en las muestras. Finalmente, con el objeto de determinar si los agrupamientos diferían significativamente según su composición foraminiferológica, se usó el análisis ANOSIM de una vía, utilizando las localidades como factor (Clarke \& Warwick 1994). Las especies tipificadoras y discriminadoras más influyentes de los grupos formados fueron identificadas con la rutina SIMPER de PRIMER.

Para el cálculo de frecuencia se usó el índice de Dajoz (1974): C = px 100 /P, donde p es el número de muestras en que se encuentra la especie y $\mathrm{P}$, el total de las muestras estudiadas. Este autor considera 3 categorías para el valor de C: 1 . Especies constantes, si están presentes en más del 50\% de las muestras, 2. Especies accesorias si están presentes en el 25 $50 \%$ de las muestras y 3 . Especies accidentales en el área si están presentes en menos del 25\% de las muestras. Por otra parte, se consideró los rangos de abundancia de Fatela (1994), quien propone que una especie puede considerarse dominante si su abundancia es > 20\% en las muestras, común si es del 10-20\%; accesoria del 5-10\% y rara, si la abundancia es del 1- 5\%.

Para comparar los foraminíferos arenáceos antárticos con los del cono sur de Sudamérica, se consideró la siguiente bibliografía: Lena (1967), Herb (1971), Earland (1932), Heron- Allen \& Earland (1932), Thompson (1978), Zapata \& Moyano (1997), Zapata \& Alarcón (1998), Violanti et al. (2000), Hromic (1996, 2002, 2011a, b y c (en prensa)), Zúñiga-Rival (2006).

\section{RESULTADOS}

\section{Composición taxonómica}

Para las tres localidades de estudio se identificó un total de 22 especies de foraminíferos del suborden Textulariina, las que se agruparon en 12 familias y 15 géneros. La Familia Trochamminidae presentó la mayor diversidad con 2 géneros y 4 especies, seguida por la familia Hormosinidae con un género (Reophax) y tres especies (Tabla 2).

Tabla 2. Sistemática del suborden Textulariina Delage y Herouard, 1886 recolectados en isla Decepción, bahía Chile y bahía Fildes, península Antártica.

\begin{tabular}{lll}
\hline \multicolumn{1}{c}{ Familia } & \multicolumn{1}{c}{ Género } & \multicolumn{1}{c}{ Especie } \\
\hline Trochamminidae & Trochammina & Trochammina malovensis Heron-Allen \& Earland, 1929 \\
& & Trochammina squamata Jones \& Parker, 1860 \\
& Deuterammina & Trochammina quadriloba Hoëglund 1948 \\
Rzehakinidae & Miliammina & Miliammina arenácea (Chapman, 1916) \\
Rhabdamminidae & Rhabdammina & Rhabdammina abyssorum Sars, 1869 \\
& Rhabdammina linearis Brady, 1879 \\
& Reophax & Rhizammina algaeformis (Brady, 1879) \\
Hormosinidae & Reophax dentaliniformis (Brady, 1884) \\
& Reophax nodulosus Brady 1884 \\
Spiroplectamminidae & Reophax pilulifer Brady, 1884 \\
Ammosphaeroidinidae & Morulaeplecta & Spiroplectammina biformis (Parker \& Jones, 1865) \\
Haplophragmoididae & Adercotryma & Morulaeplecta bulbosa Höglund 1947 \\
& Labrospira & Adercotryma glomerata (Brady, 1878) \\
Eggerellidae & Labrospira jeffreysii (Williamson, 1858) \\
Cyclamminidae & Labrospira kosterensis (Hoeglund, 1947) \\
Pseudobolivinidae & Eggerella minuta (Wiesner, 1931) \\
Saccamminidae & Alveolophragmiun & Alveolophragmiun subglobossum (Sars, 1868) \\
Textulariidae & Plveolophragmiun columbiense (Cushman, 1925) \\
\hline
\end{tabular}




\section{Riqueza específica}

El número promedio de especies de foraminíferos arenáceos identificados fue de 4,41 especies por muestra, considerando el total de muestras analizadas en las tres localidades y en los tres períodos de muestreo.

En las 16 muestras recolectadas en isla Decepción, se identificó un total de 13 especies (59,1\% del total identificado para toda el área de estudio) correspondiendo a un promedio de 3,9 especies por muestra. En tres de ellas se recolectó sólo una sola especie, siendo el número máximo identificado de 7 especies. En las 20 muestras obtenidas en bahía Chile, se identificaron 20 especies (90,9\% del total), con un promedio de 5,1 especies por muestra. En una de ellas se obtuvo solo una especie, siendo el máximo de 10 especies. En bahía Fildes, se estudiaron 13 muestras e identificaron 13 especies (59,1\% del total), con un promedio de 4,5 especies por muestra. En dos de ellas se encontró solo una especie, siendo el máximo identificado de 9 especies (Fig. 2).

Las especies con mayor abundancia en el área fueron Trochammina malovensis, claramente dominante (> 50\%), Miliammina arenácea (especie común) (15\%) y Reophax dentaliniformis (especie accesoria) (5\%). Alrededor de un tercio $(36,4 \%)$ de las especies de textuláridos superaron el $2 \%$ de abundancia, el resto de especies (63,5\%) mostró escasa representación y pueden considerarse raras (Fatela 1994) (Fig. 3).

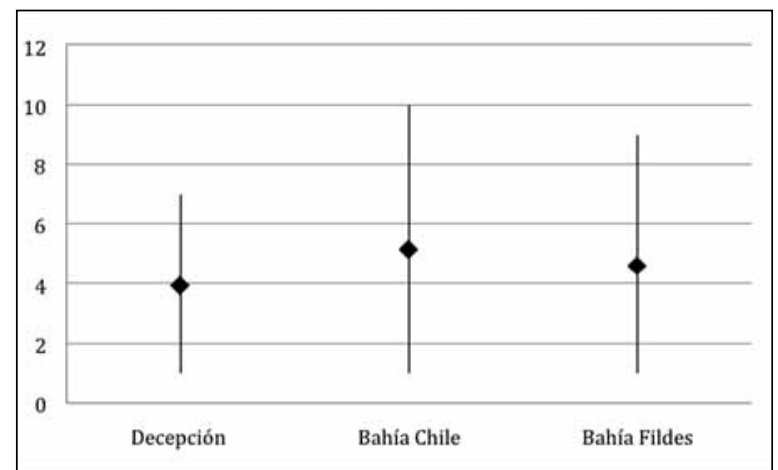

Fig. 2. Número promedio de especies de textuláridos identificados para las tres áreas y en los tres períodos de estudio. (Decepción $n=16$, báhía Chile $n=20$ y bahía Fildes $n=13$ especies).

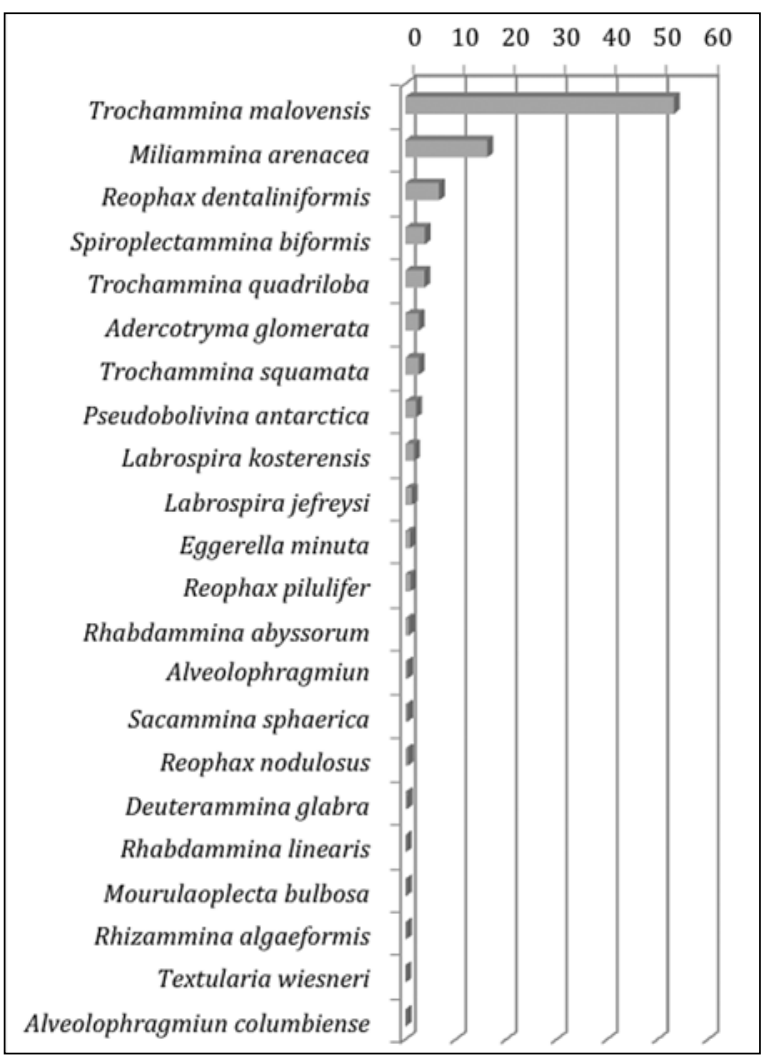

Fig. 3. Abundancia relativa de las especies de Textulariina presentes en aguas someras $(<50 \mathrm{~m}$ ) de Decepción bahía Fildes y bahía Chile, península antártica.

\section{Abundancia}

Se recolectó un total de 2.573 ejemplares, el $57 \%$ de ellos fueron extraídos en isla Decepción, el $26 \%$ en bahía Chile y el $17 \%$ en bahía Fildes. El número promedio de foraminíferos arenáceos en isla Decepción fue de 91 ejemplares/ muestra, mostrando una gran heterogeneidad y siendo el área con mayor número de foraminíferos. En bahía Chile, se obtuvo 25 ejemplares y en bahía Fildes, 26 ejemplares promedio /muestra, siendo las abundancias más homogéneas. La abundancia según localidad se muestra en la figura 4.

\section{Frecuencia}

Las especies que alcanzaron la mayor distribución geográfica, fueron Trochammina malovensis (presente en el 95,9\% de las muestras), Miliammina arenacea (73,5\%), Spiroplectammina biformis 


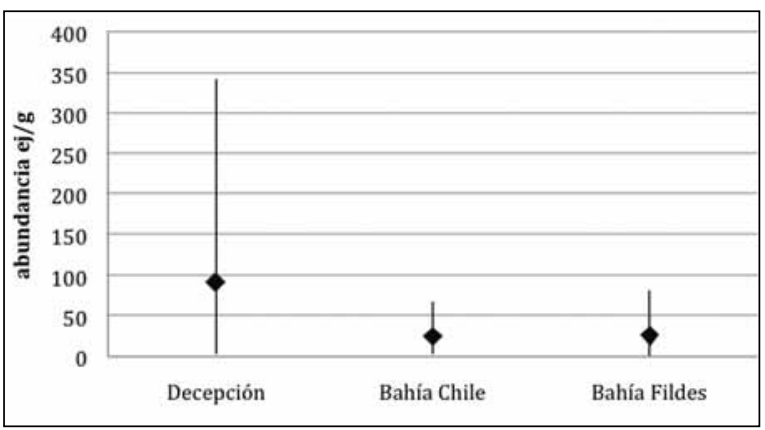

Fig. 4. Abundancia según localidad.

(63,3\%) y Trochammina squamata $(61,2 \%)$, que se recolectaron en más de la mitad de las estaciones y por los tanto pueden considerarse constantes en el área de acuerdo al Índice de Dajoz. Como especies accesorias se encontrarían Adercotryma glomerata (44,9\%), Pseudobolivina antárctica (38,8\%), Reophax dentaliniformis $(40,8 \%)$ y Labrospira jeffreysii (36,7\%). El resto de los textuláridos (14 especies) pueden considerarse como especies accidentales $(<22,5 \%)$ (Fig. 5).

\section{Aspectos comunitarios}

El análisis de similitud y SIMPROF permitió visualizar la tendencia a la conformación de 3 grupos (Fig. 6), lo que se verificó con el análisis MDS (estres $=0,2$ ) (Fig. 7). Sin embargo éstos fueron muy heterogéneos con respecto a la localidad de procedencia de las muestras, y no mostraron diferencias significativas.

El grupo 1, reunió la mayoría de las muestras (34 muestras), extraídas indistintamente desde las tres localidades: Fildes (12), Bahía Chile (12) e isla Decepción (11). Se separó de este este grupo una muestra obtenida en bahía Chile ( $\mathrm{CH}$ st.1). El grupo 2 , incluyó un total de 10 muestras, cinco de ellas procedentes de Decepción (d2, d4, d5, d6 y de5), cuatro procedentes de bahía Fildes (f2, f3, f4 y Fi4) y una extraída en bahía Chile (chi1). El grupo 3, estuvo formado por 4 muestras, tres de llas extraídas en la bahía Chile (Ch2, Ch6 y chi 7) y una muestra extraída en la bahía Fildes (Fi3). No se incluyó en este grupo muestras procedentes de isla Decepción. Se

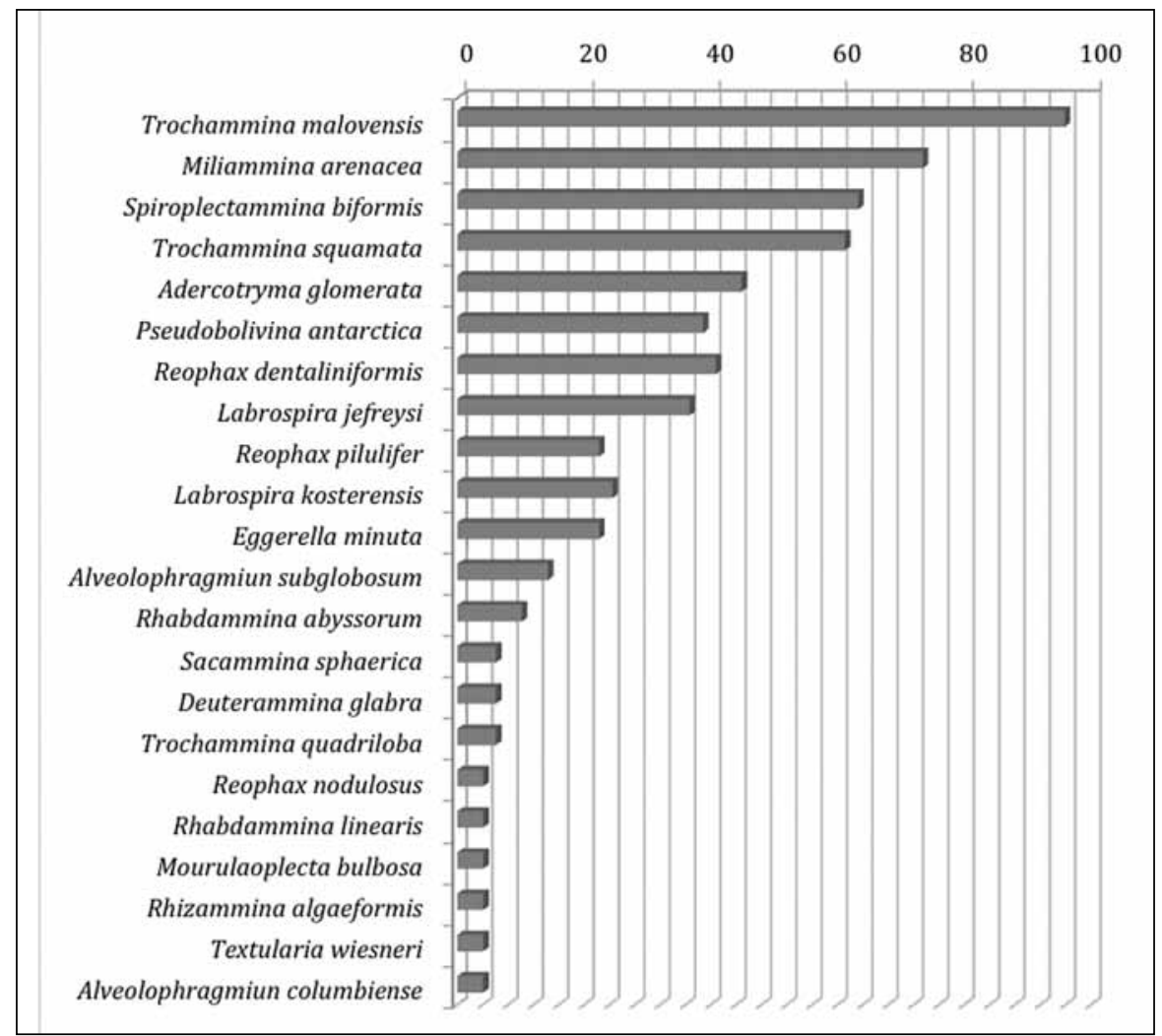

Fig. 5. Distribución geográfica de la especies de Textulariina presentes en aguas someras $(<50 \mathrm{~m})$ en la península antártica. 


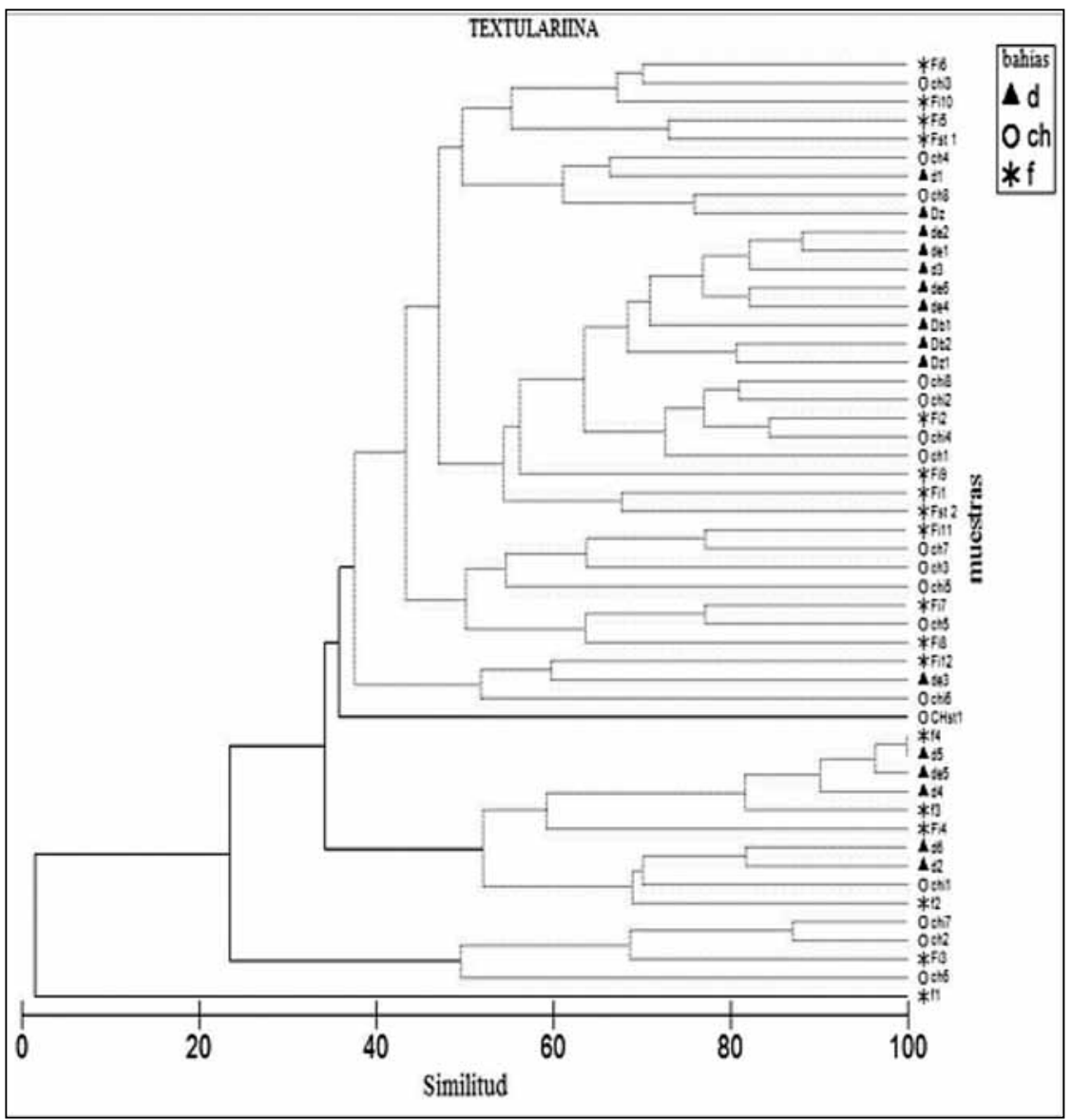

Fig. 6. Dendrograma (Índice de Bray-Curtis) y SIMPROF, de las muestras extraídas en la península antártica (bahía Fildes (f), bahía Chile (ch), isla Decepción (d). Los símbolos identifican las bahías en que fueron recolectadas las muestras.

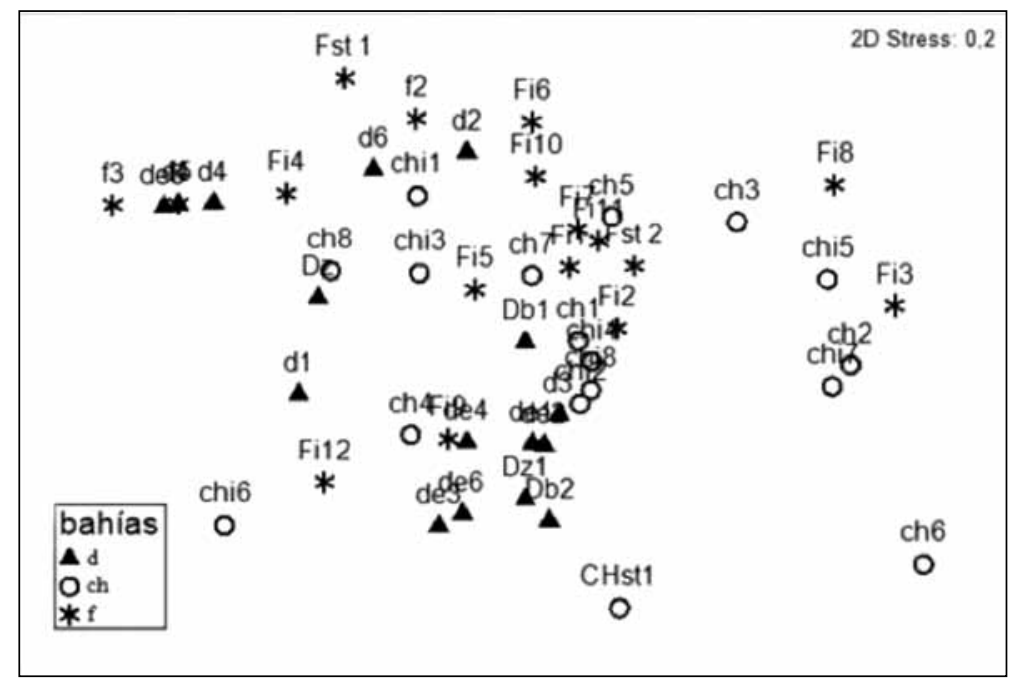

Fig. 7. Diagrama de ordenación (MDS). Los símbolos corresonden a la bahía desde la que se extrajeron las muestras: Decepción (d), bahía Fildes (f) y bahía Chile (ch). 
separa del los grupos significativamente la muestra f1 obtenida en bahía Chile.

Respecto de la similitud faunística en cada una de las localidades, los foraminíferos de la isla Decepción mostraron un porcentaje de similitud del 50,6\% y las especies que más contribuyeron al agrupamiento fueron Trochammina malovensis (67\%), Miliammina arenácea (11,1\%), Spiroplectammina biformis (8,6\%) y Reophax dentaliniformis (8,5\%). La fauna de bahía Chile mostró un porcentaje de similitud del $41,7 \%$ y las especies tipo fueron: Miliammina arenácea (23,2\%), Trochammina malovensis (22,7\%), Trochammina squamata (17\%), Spiroplectammina biformis (15\%), Adercotryma glomerata (7\%) y Pseudobolivina antárctica $(5,4 \%)$. Finalmente la fauna de bahía Chile Presentó un porcentaje de similitud del 36,4\% y las especies que más contribuyeron a dicha similitud fueron: Trochammina malovensis (51,4\%), Miliammina arenacea $(13,8 \%)$, Trochammina squamata $(9,5 \%)$, Adercotryma glomerata (8,2\%), Labrospira jeffreysii $(4,4 \%)$ y Reophax pilulifer $(4,4 \%)$.

No se observaron diferencias significativas en la composición de foraminíferos entre las tres bahías, el análisis ANOSIM entregó un R de 0,126; p=0,002, por lo cual se acepta la hipótesis nula, que no hay diferencias significativas entre las comunidades.
El análisis SIMPER permitió definir también las especies discriminadoras. Si bien, T. malovensis, Miliammina arenácea y $T$. squamata están presentes en prácticamente todos los grupos sus diferentes abundancias parecen explicar la discriminación observada. En la tabla 3 se muestra las especies responsables de la disimilitud.

Relación con foraminíferos arenáceos del área subantártica

La revisión bibliográfica permitió detectar que cerca del 50\% de las especies determinadas para Antártica, no se registran en el continente sudamericano, costa pacífica, al sur de $42^{\circ} \mathrm{S}$. De las 22 especies identificadas en las aguas antárticas $(<50 \mathrm{~m})$ las siguientes 10 especies no han sido citadas en la región austral chilena del continente sudamericano: Alveolophragmiun columbiense, Deuterammina glabra, Eggerella minuta, Pseudobolivina antárctica, Reophax nodulosus, Rhabdammina linnearis, Saccammina sphaerica, Textularia wiesneri, Trochammina malovensis y T. quadriloba, por lo que podrían considerarse endémicas para la región antártica.

Tabla 3: Relación entre grupo, porcentaje de disimilitud y especies discriminadoras con sus respectivos porcentajes de contribución de las agrupaciones formadas según SIMPER (PRIMER 6.0). En la última columna se muestra el porcentaje de contribución acumulado. (d) Isla Decepción; (ch) bahía Chile; (f) bahía Fides.

\begin{tabular}{|c|c|c|c|c|}
\hline Grupos & $\%$ disimilitud & especies & $\%$ contribución & $\%$ acumulado \\
\hline d y ch & 60,1 & $\begin{array}{l}\text { Trochammina malovensis } \\
\text { Miliammina arenácea } \\
\text { Reophax dentaliniformis } \\
\text { Trochammina squamata } \\
\text { Spiroplectammina biformis } \\
\text { Adercotryma glomerata } \\
\text { Pseudobolivina antarctica }\end{array}$ & $\begin{array}{l}19,8 \\
15,3 \\
11,3 \\
10,4 \\
10,3 \\
7,2 \\
6,8\end{array}$ & $\begin{array}{l}19,8 \\
35,1 \\
46,2 \\
56,6 \\
66,9 \\
74,1 \\
80,9\end{array}$ \\
\hline$d y f$ & 59,8 & $\begin{array}{l}\text { Trochammina malovensis } \\
\text { Miliammina arenácea } \\
\text { Reophax dentaliniformis } \\
\text { Spiroplectammina biformis } \\
\text { Trochammina squamata } \\
\text { Reophax pilulifer }\end{array}$ & $\begin{array}{l}15,3 \\
14,9 \\
9,8 \\
9,8 \\
6,2 \\
5,8\end{array}$ & $\begin{array}{l}15,3 \\
30,2 \\
40,9 \\
50,7 \\
79,8 \\
85,7\end{array}$ \\
\hline $\operatorname{ch} y \mathrm{f}$ & 60,1 & $\begin{array}{l}\text { Trochammina malovensis } \\
\text { Miliammina arenácea } \\
\text { Spiroplectammina biformis } \\
\text { Trochammina squamata } \\
\text { Afercotryma glomerata } \\
\text { Pseudobolivina antractica } \\
\text { Labrospira jeffreysii } \\
\text { Reophax pilulifer }\end{array}$ & $\begin{array}{l}14,7 \\
12,2 \\
10,4 \\
10,3 \\
8,7 \\
8,4 \\
7,2 \\
5,5\end{array}$ & $\begin{array}{l}14,7 \\
26,9 \\
37,3 \\
47,6 \\
56,4 \\
64,7 \\
71,9 \\
77,3\end{array}$ \\
\hline
\end{tabular}


Tabla 4. Relaciones de las especies de textuláridos encontradas en la península antártica con las especies del cono sur de América. 1.a) Zapata \& Alarcón (1988); b) Violanti et al. (2000), c) Hromic (1996); 2. Lena (1967); 3. Hromic (2002); 4. Zúñiga (2006); 5. a)Hromic \& Zúñiga (2005), b) Boltovskoy \& Totah (> 45ํ) (1987); 6. Zapata \& Moyano (1997); 7. Thompson (1978); 8. Earland (1932); 9. a)Hromic (2011 a), b) Hromic (2001).

\begin{tabular}{|c|c|c|c|c|c|c|c|c|c|}
\hline & 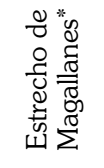 & 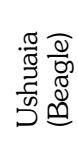 & 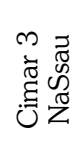 & 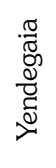 & 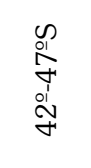 & 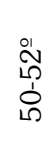 & 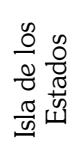 & $\begin{array}{l}\infty \\
\infty \\
m \\
\dot{\omega}\end{array}$ & 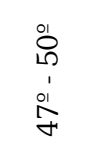 \\
\hline & 1 & 2 & 3 & 4 & 5 & 6 & 7 & 8 & 9 \\
\hline Adercotryma glomerata & & & & & $\mathrm{Xb}$ & & & & \\
\hline \multicolumn{10}{|l|}{ Alveolophragmiun columbiense } \\
\hline Alveolophragmiun subglobosum & $\mathrm{Xb} \mathrm{Xc}$ & & & & & & & & \\
\hline \multicolumn{10}{|l|}{ Deuterammina glabra } \\
\hline \multicolumn{10}{|l|}{ Eggerella minuta } \\
\hline Labrospira jeffreysii & $\mathrm{Xb}$ & & $\mathrm{X}$ & & Хa & & $\mathrm{X}$ & & $\mathrm{Xb}$ \\
\hline Labrospira kosterensis & & & $\mathrm{X}$ & & $\mathrm{Xa}$ & & & & $\mathrm{XaXb}$ \\
\hline Miliammina arenacea & & & & $\mathrm{X}$ & $\mathrm{Xa}$ & & & & \\
\hline Morulaeplecta bulbosa & $\mathrm{Xa}$ & & & & & & & & \\
\hline \multicolumn{10}{|l|}{ Pseudobolivina antarctica } \\
\hline Reophax dentaliniformis & & & $\mathrm{X}$ & & & $\mathrm{X}$ & & & \\
\hline \multicolumn{10}{|l|}{ Reophax nodulosus } \\
\hline Reophax pilulifer & $\mathrm{Xb} \mathrm{Xc}$ & & $\mathrm{X}$ & & & $\mathrm{X}$ & $\mathrm{X}$ & & \\
\hline Rhabdammina abyssorum & $\mathrm{Xb}$ & & $\mathrm{X}$ & & & & & & \\
\hline \multicolumn{10}{|l|}{ Rhabdammina linearis } \\
\hline Rhizammina algaeformis & & & & & & & & & $\mathrm{XaXb}$ \\
\hline \multicolumn{10}{|l|}{ Sacammina sphaerica } \\
\hline Spiroplectammina biformis & & $\mathrm{x}$ & $\mathrm{x}$ & & $\mathrm{XaXb}$ & & $\mathrm{x}$ & & $\mathrm{Xb}$ \\
\hline \multicolumn{10}{|l|}{ Textularia wiesneri } \\
\hline \multicolumn{10}{|l|}{ Trochammina malovensis } \\
\hline \multicolumn{10}{|l|}{ Trochammina quadriloba } \\
\hline Trochammina squamata & & & & & $\mathrm{X}$ & & & & \\
\hline
\end{tabular}

\section{DISCUSIÓN}

De acuerdo con Hromic (19993) los foraminíferos arenáceos corresponden al 44,9\% de las especies y al $48 \%$ del total de los ejemplares recolectados durante estas expediciones (ECA XXXII, XXXIII y XXXIV). Estos porcentajes son mucho más altos que los observados en las costas del cono sur de América, Chile, en donde los textuláridos constituyen ca. del 10\% de los ejemplares (Hromic 2011a, b, y d (en prensa)). Recientemente Hromic \& Montiel (2011) dan a conocer que este aumento de textuláridos se presenta también en ambientes periglaciales de la región austral chilena, en donde la abundancia llega al 45,8\%.

3 Hromic, T. 1999. Informe final Proyecto "Los foraminíferos bentónicos antárticos y sus vinculaciones con la microfauna sudamericana" INACH.
Lena (1975) revisó una veintena de muestras, obtenidas en el sector de la isla Elefante (Antartida) extraídas desde profundidades entre $10-800 \mathrm{~m}$, obteniendo resultados algo diferentes. De las 5 muestras extraídas a $<63 \mathrm{~m}$, en 3 no obtuvo foraminíferos y en 2 , se contaron escasísimas especies de textuláridos ( $\mathrm{N}^{\circ}$ 228: 2 arenáceos; $\mathrm{N}^{\circ}$ 229: 4 arenáceos). En general, Lena encontró que en este sector, el número de especies y géneros calcáreos fue mayor que el de los arenáceos y entre estos últimos las especies más abundantes fueron: A. glomerata, C. jeffreysii (raro $<63 \mathrm{~m}$ ), M. arenacea (rara $<63$ $\mathrm{m}), R$. dentaliniformis, $T$. squamata $f$. astrifica (frecuente $<63 \mathrm{~m}$ ) y $T$. weisneri (frecuente a $<63$ $\mathrm{m})$. Esta autora señala que el número máximo de foraminíferos se registró en 0-200 m, los que luego disminuyen para volver a aumentar bajo los $600 \mathrm{~m}$. Establece como un primer límite batimétrico, basada 
en foraminíferos vivos, los $130 \mathrm{~m}$, caracterizado por el domino de especies arenáceas.

Posteriormente Lena (1980) informó que en la península antártica el $40 \%$ de las especies y el $72,5 \%$ de los ejemplares eran formas arenáceas, lo que difiere de los resultados obtenidos en este trabajo y que puede atribuirse a que esta autora trabajó muestras extraídas desde profundidades entre 10 y $1.030 \mathrm{~m}$. Esta autora concuerda en la presencia de A. glomerata, $C$. jeffreysii, $M$. arenacea, $P$. antárctica, $S$. biformis, $R$. pilulifer y $T$. weisneri y entrega una lista de otros arenáceos no recolectados en este estudio y que podría relacionarse también con la profundidad. Al parecer T. malovensis es reemplazada por Trochammina antarctica que alcanza el 29,3\% de los ejemplares, y Trochammina squamata f. astrifica, el 25,7\%, siendo esta última la de mayor distribución, en 85,7\% de las muestras. Como acompañantes T. discorbis Earland, T. inflata (Montagu), T. weisneri Parr y Pseudobolivina antárctica el 5,7\%.

Martínez \& Martínez (1994) identificaron para bahía Chile dos sectores, en el primero, interno, dominan los foraminíferos calcáreos, principalmente del género Pyrgo; y entre los arenáceos Trochammina nana (Brady) (5\%) y L. jeffreysii (1,5\%), En el segundo sector, externo, también dominan representantes de Pyrgo, los que se encuentran acompañados de $R$. pilulifer (1,5\%). Es posible que estas diferencias en la composición faunística se deba a variaciones interanuales normales, con proliferación de especies oportunistas. McKnight (1962) menciona que en antártica muestras obtenidas desde la misma profundidad pueden estar bajo condiciones oceanográficas muy distintas y por ende, contener fauna muy diversa.

Trochammina es un género de amplia distribución; se le encuentra de $0-30^{\circ} \mathrm{C}$ y de 0 a $>$ $6.000 \mathrm{~m}$ de profundidad, en aguas templadas - frías y con salinidades de $0-60 \%$. Puede adaptarse a la vida infaunal- epifaunal (Murray 1991). Su gran diversidad en Antártica, ha sido mencionada por diferentes autores. La dominancia de Trochammina malovensis en sedimentos someros de la isla Rey Jorge fue dada a conocer por Li \& Zhang (1986, fide Murray 1991). Finger \& Lipps (1981) señalan que esta especie es polimórfica y que posiblemente formas como $T$. squamata, $T$. intermedia y $T$. astrifica y constituyan un solo taxa, lo que sugiere que este género tendría solo unas pocas especies, con varios ecotipos.

Miliammina, por su parte es un taxa con hábitos exclusivamente infaunales, capaz de adaptarse a distintos ambientes. Reophax, es un género también infaunal, pero claramente marino, restringudo a aguas temperado - frías (Murray, 1991). Esto explicaría las variaciones faunísticas acordes a las variaciones estacionales de Antártica. Otras especies de textuláridos registradas en antártica de manera esporádica son R. linnearis (Mar de Weddell), T. wiesneri (Mar de Scotia), P. antárctica (islas Orcadas, McMurdo y mar de Ross y Sandwich del Sur), A. glomerata (Sandwich del Sur), R. pilulifer (MacMurdo, pasaje Drake), D. glabra (Mc Murdo) todas propias de zonas más profundas (Murray 1991). Cabe destacar que $R$. pilulifer se define como una especie típica de las Shetland del Sur (Herb, 1971).

Finger \& Lipps (1981) estudiaron los foraminíferos bentónicos por 5 años consecutivos en la isla Decepción tanto en su interior como en la plataforma externa. En el interior registraron el dominio de Miliammina arenacea y T. malovensis, lo que concuerda con este estudio y en el exterior, Labrospira jeffreysii (=Cribrostomoides jeffreysii), y Lepidodeuterammina ochracea (= Rotaliammina ochracea).

Mateu (1989) estudio 67 muestras obtenidoas en Antartica encontrando en el área 1 (Isla Elefante, Shetland del Sur, Isla Decepción, Isla del Rey Jorge) entre 80- $340 \mathrm{~m} R$. scorpiurus, $R$. fusiformis y $R$. dentaliniformis.

Herb (1971) estudió 73 muestras en la zona del Paso Drake todas extraídas a altas profundidades. En las muestras recolectadas en $73 \mathrm{~m}$ y $104 \mathrm{~m}$, al sur de la convergencia antártica registró la presencia de las siguientes especies de textuláridos: Reophax pilulifer, $R$. nodulosus, L. jeffreysii, Bathysiphon filiformis, Rhizammina indivisa y Saccammina sphaerica. Al norte de la convergencia además de estas especies, encontró Reophax nodulosus y Cyclammina pusilla. Este autor intenta una zonación, señalando para la plataforma interna la presencia de Trochammina aff. T. squamata y L. jeffreysii. También muestra como los foraminíferos arenáceos tienen mayor presencia en la península antártica en comparación al Cabo de Hornos, donde comienzan a predominar los organismos calcáreos. Estos resultados concuerdan plenamente con los obtenidos en este trabajo. 
Hromic \& Zúñiga-Rival $\left(2003^{4}\right)$ revisaron 7 muestras ( $400 \mathrm{~m})$ extraídas en el Arco de Scotia en las cuales, el 77,4 \% fueron foraminíferos arenáceos. Las principales especies recolectadas por estas autoras fueron M. arenacea (23,1\%), Reophax pilulifer (13,5\%), Trochammina antárctica (12,7\%), Bathysiphon sp. (10,7\%), lo que sugiere que los foraminíferos arenáceos, llegarían a Sudamérica a través del arco de Scotia y que en profundidades más altas Trochammina malovensis podría ser reemplazada por $T$. antárctica.

Los foraminíferos arenáceos disminuyen en latitudes más bajas, sobre el continente sudamericano. En bahía Nassau, Cabo de Hornos, constituyen el $0,4 \%$ del total de los especímenes y solo están representados por 4 especies, L. jeffreysii, L. kosterensis, L. ochracea y Siphotextularia sp., en cantidades $<1 \%$ (Hromic 2002). En la costa de Ushuaia Lena (1967), registró abundantes A. columbiense (= Cribrostomoides columbiense Cushman var. robustum (Cushman \& McCulloch)) y además de mayor tamaño $(1,41 \mathrm{~mm})$. También encontró Haplophragmoides hancoki Cushman \& McCulloch, frecuente en aguas hiposalinas; Saccammina atlántica (rara) y $S$. decorata (frecuente). A $9 \mathrm{~m}$ de profundidad, una de las especies más frecuentes fue Spiroplectammina biformis (ejemplares vivos) la que se encontraba acompañada de abundantes formas de T. plana discorbis Earland y T. squamata Jones \& Parker. Esta autora señaló que la relación calcáreo/ aglutinado es 27\%. En la bahía Yendegaia (5440' S-68 $50^{\prime}$ W), Miliammina arenacea fue recolectada en el ensamble intermareal con una abundancia del 16,8\% (Zúñiga 2006).

En el estrecho de Magallanes los textuláridos constituyen el 2,2\% de los ejemplares y están representados por varias especies: A. subglobossum. $L$. jeffreysii, R. pilulifer, C. nítida, H. bradyi, H. globigeriniformes, Martinotiella communis, y R. scitulum (Hromic 1996). Las tres primeras se encuentran en la península Antártica y son cosmopolitas. Otras especies presentes en el estrecho de Magallanes son $H$. canariensis. A. incertus, $C$. jeffreysii, $C$. subglobossus, $H$. friabilis, M. nodulosa, $R$. scor-

4 Hromic, T. \& M. Zúñiga-Rival 2003. Scotia Arc, a bridge between Antarctic and South America microfauna distribution? Biological Interaction between the Antarctic and the Magellan Region (IBMANT II) Symposium \& Workshop, October 2003, Ushuaia, Argentina. piurus y trocamínidos. Señalan que $R$. pilulifer es una especie común (5,96\%), en la muestra $57 \mathrm{~V}$ cerca del Cabo Froward, en donde los foraminíferos arenáceos tienen su máxima abundancia (Muestras $57 \mathrm{~S}$ y $57 \mathrm{~V})$, también son abundantes en la cuenca profunda occidental (muestras $40 \mathrm{~V} 54 \mathrm{RV}$ ) (Violanti et al. 2000). Zapata \& Alarcón (1988) encontraron en este mismo sector, Ammodiscus flavidus Höglund, Cribrostomoides hancocki (Cushman \& McCulloch), Morulaeplecta bulbosa (Höeglund), Remaneica helgolandica Rhumbler, Trochammina inflata (Montagu), T. ochcracea (Williamson) y T. plana Egger. Otros trabajos (Marchant 1993, Hromic 2010) a pesar de dar a conocer fauna de ambientes someros, (bahía Scholl y fiordo Comau respectivamente), no citan especies arenáceas.

\section{CONCLUSIÓN}

Los foraminíferos arenáceos están representados en los sedimentos someros antárticos (<50 m) por 12, familias, 15 géneros y 22 especies. Estos foraminíferos se encuentran formando el 44,9\% de las especies y el $48 \%$ de los foraminíferos bentónicos. La biodiversidad en las muestras es muy variada, desde 1 a 10 especies siendo el promedio más alto en bahía Chile, en la cual se encontraron representadas el 90\% de las especies. Las especies con mayor abundancia en el área fueron Trochammina malovensis, Miliammina arenacea y Reophax dentaliniformis. En isla Decepción se recolectó la mayor parte de los ejemplares (57\%). Las especies que alcanzaron la mayor distribución geográfica, fueron Trochammina malovensis (presentes en el 95,9\% de las muestras), Miliammina arenácea (73,5\%), Spiroplectammina biformis $(63,3 \%)$ y Trochammina squamata (61,2\%). El análisis estadístico no brindó diferencias significativas entre las tres localidades estudiadas (bahía Chile, bahía Fildes e isla Decepción), lo que permite concluir que se trata de una sola comunidad faunística relativamente homogénea, sobre la cual incidirían las variaciones locales, que afectarían principalmente a la abundancia de las especies de los sedimentos someros.

En relación a su vinculación con los textuláridos del área sudamericana, alrededor del 50\% de las especies identificadas en los sedimentos someros antárticos se encuentran también en el área subantártica sudamericana. Se considera que 
las siguientes especies son endémicas para la zona antártica: Alveolophragmiun columbiense, Deuterammina glabra, Eggerella minuta, Pseudobolivina antárctica, Reophax nodulosus, Rhabdammina linnearis, Saccammina sphaerica, Textularia wiesneri, Trochammina malovensis y T. quadriloba.

\section{AGRADECIMIENTOS}

Al Instituto Antártico Chileno, por el financiamiento del Proyecto "Foraminíferos recientes antárticos y sus vinculaciones con la microfauna sudamericana", a la Dirección de Investigación de la Universidad de Magallanes por el financiamiento del Proyecto "Foraminíferos textuláridos de canales y fiordos chilenos" y al Instituto de la Patagonia de la Universidad de Magallanes por las facilidades para su desarrollo.

Se agradece muy especialmente al Dr. Américo Montiel y al Dr. Carlos Ríos, por sus valiosos consejos en el análisis estadístico.

\section{LITERATURA CITADA}

Arntz, W., G.A. Lovrich \& S. Thatje 2005. The Magellan-Antartic connection: Links and frontiers at high southern latitudes. Scientia Marina, 69 (Supl. 2):373 pp.

Barker, R.W. 1960 Taxonomic notes on the species figured by H. B. Brady in his "Report on the Foraminifera dredged by H. M. S. Challenger during the years 1873-1876". Society Economic Paleontological \& Mineral Special Pub. $\mathrm{N}^{\circ}$ 9; Oklahoma USA, 238 pp.

Boltovskoy, E. 1965. Los Foraminíferos Recientes. Eudeba. Bs. Aires. 510 pp.

Boltovskoy, E \& V. Totah 1987. Relación entre masas de agua y foraminíferos bentónicos en el Pacífico sudoriental. Physis (Bs. Aires) Sección A., 45 (109):37-46

Boltovskoy, E., G. Giussani, S. Watanabe \& R. Wrigth 1980. Atlas of benthic shelf foraminifera of the southwest atlantic Junk. bv. Pub. The Hague -Boston- London. 147 pp.

Boschi, E.E. \& M. Gavio. 2005. On the distribution of decapod crustaceans from the Magellan biogeographic provinces and the Antarctic region. Scientia Marina, 69 (Suppl. 2):195200
Brady, H. B. 1884. Report on the foraminifera dredged by H. M. S. Challenger during the years 1873 - 1876. Reports of the scientific results of the voyage of $H$. M. S. Challenger (Zoology). 9:1-800

Clarke, K. \& R. Gorley 2006. Primer v.6 User Manual/Tutorial PRIMER-E Ltd.: Plymouth Marine Laboratory. UK. 190 pp.

Clarke, K. R. \& R.M. Warwick 1994. Change in marine communities: an aproach to statistical analysis and interpretation. Plymouth Marine Laboratory.

Dajoz, R. 1974. Tratado de Ecologia. Ed. MundiPrensa. $478 \mathrm{pp}$.

Decrouez, D. 1989. Generic ranges of Foraminiferida. Revue Paleobiologie, 8 (1):263-321

Earland, A. 1933. Foraminifera. Part II. South Georgia. British Antarctic Expedition.Discovery Report, VII:27-138. Pl. I-VII.

Earland, A. 1934. Foraminifera Part III. The Falklands sector of the Antartic (excluding South Georgia). Discovery Report. X: 1 208. Pl. I - X.

Ellis, B. \& A. Messina 1940 (et seq.). Catalogue of Foraminifera. Am. Museo of Natural History. Special Pub. 30 volúmenes.

Fatela, F. 1994. Contribution dés foraminifères bentiques profunds à la reconstitution des apléoenvironments du Quaternaire récent de la marge Ouest Ibérique (marge Nord Portugaise, Banc de Galice) Thèse de doctorat, Univ. Bordeaiux I, Freancia 241 pp.

Finger, K.L. \& J.H. Lipps 1981. Foraminiferal decimation and repopulation in an active volcanic caldera Deception Island, Antarctica. Micropaleontology, 27 (2):111-139

Herb, R. 1971. Distribution of recent benthonic foraminifera in the Drake Passage. Antarctic Research Serie 17:251-300.

Hermelin, J.O. 1983. Biogeographic patterns of Modern Reophax dentaliniformis Brady (arenaceous benthonic foraminifera) from the Baltic Sea. Journal of Foraminiferal Research, 13 (3):155-162

Heron-Allen, E. \& A. Earland 1932. Protozoa, Part II Foraminifera. British Antarctica (Terra Nova) Expedition, 1910, Natural History Report, Zoology,6 (2):25-268 
Höglund, H. 1947. Foraminifera in the Gullmar Fjord and the Skagerak. Zoologiska Bidrag Fran Uppsala, 26:1-328

Hromic, T. 1996. Foraminíferos bentónicos (Protozoa: Foraminiferida) de aguas profundas del estrecho de Magallanes, Chile. Anales Instituto Patagonia, Ser. Cs. Nats. (Chile), 24:65-86

Hromic, T. 2001. Foraminíferos bentónicos del canal Baker ( $\left.47^{\circ} \mathrm{S} ; 74^{\circ} \mathrm{W}\right)$ Pacífico sudoriental, Chile Anales Instituto Patagonia Ser. Cs. Nat. (Chile), 29:135-156.

Hromic, T. 2002. Foraminíferos bentónicos de Bahía Nassau, Cabo de Hornos, Chile. Comparación con foraminíferos del cono sur de América, Antártica e islas Malvinas. Anales Instituto Patagonia, Ser. Cs. Nat. (Chile), 30:95-108

Hromic, T. 2009 a. Estructura comunitaria del taxa Foraminiferida (Protozoa) según profundidad, en el fiordo Comau, Chiloé, Chile. Anales Instituto Patagonia (Chile), 37 (1):39-51

Hromic, T. 2011 a. Foraminíferos bentónicos recolectados durante la expedición Cimar 14 Fiordos, Patagonia chilena. Anales Instituto Patagonia (Chile), 39 (1):75-89

Hromic, T. 2011b (en prensa). Análisis de la comunidad foraminiferológica bentónica del seno Reloncaví, islas Desertores, golfo Ancud y golfo Corcorvado, Chile. Revista de Ciencia y Tecnología del Mar 34 (1)

Hromic, T. 2011c. Foraminíferos bentónicos recientes del sur del estrecho de Magallanes, Expedición Cimar 3 Fiordos. Canales australes chilenos (52-56오. Anales Instituto Patagonia (Chile), 39 (2):17-32

Hromic, T. 2011d (en prensa). Foraminíferos bentónicos de la expedición Cimar 11 Fiordos, canales patagónicos chilenos: biodiversidad y abundancia. Revista de Ciencia y Tecnología del Mar, 34 (2).

Hromic, T. \& A. Montiel 2011. Foraminíferos bentónicos de seno Gallegos y bahía Brookes $\left(54,5^{\circ}\right.$ S-69, $\left.5^{\circ} \mathrm{S}\right)$, Chile: Patrones de distribución y diversidad. ). Anales Instituto Patagonia, 39 (2):33-46

Hromic, T. \& M. Zúñiga 2005. Foraminíferos bentónicos de ambientes someros extraídos durante la expedición Cimar 7 fiordos, canales patagónicos $\left(42^{\circ}-47^{\circ} \mathrm{S}\right)$. Boletín de la Sociedad de Biología de Concepción, 76:25-38
Hromic, T., S. Ishman \& N. Silva 2006. Benthic foraminiferal distribution in Chilean fjords: $47^{\circ} \mathrm{S}$ to $54^{\circ} \mathrm{S}$ Marine Micropaleontology, 59:115-134

Kaiho, K. 1994. Benthic foraminiferal dissolved oxygen indexand disolved oxygen levels un the modern ocean. Geology, 22:719-722

Lena, H. 1967. Foraminíferos recientes de Ushuaia (Tierra del Fuego, Argentina). Ameghiniana, 4 (9):311-336

Lena, H. 1975. Foraminíferos bentónicos del área de isla Elefante (Antártica). Physis, Secc. A, 34 (89):405-431

Lena, H. 1980. Foraminíferos bentónicos del noroeste de la Península Antártica Physis, Secc. A, 39 (96):9-20

Li \& Zhang In: Murray, J. 1991 Ecology and Palaeoecology of Benthic foraminifera. Longman Scientific Technical.UK. 397 pp.

Loeblich, A. \& H. Tappan 1988. Foraminiferal Genera and Their Classification. Van Nostrand Reinhold Co. N. Y. Text- Vol: 970, Pl-Vol. $212 p+847$ pp.

Majewski, W. 2010. Benthic foraminifera from west Antarctic fiord environments: an overview. Polish Polar Research, 31 (1):61-82

Martínez, G., R. \& R. P. Martínez 1994. Foraminíferos bentónicos recientes de Bahía Chile, Isla Greenwich, Islas Shetland del Sur. Su significado para la historia paleoclimática holocena de la Antártica Occidental. $7^{\circ}$ Congreso Geológico Chileno. Universidad de Concepción, Chile: 1689-1692. Actas

Mateu, G. 1989. Micropaleontología sedimentaria. Informe de resultados de la campaña "Antartida 8611". Publicación Especial del Instituto Español de Oceanografía, 1ª Exp. Cient. Pesquera en la Antartida 1986-1987. Sec. Gral. de Pesca Marítima M. A. P. A. Min. Agric., Pesca y Alim. Madrid, 2:83-173 Marchant, M. 1993. Foraminíferos de la Bahía Scholl, Región Magallánica, Chile, (Protozoa: Foraminifera). Gayana Zoology, 57 (1):61-75

Mc Knight, W. 1962. The distribution of foraminifera of parts of the Antarctic coast. Bulletin American Paleontolgy, 44 (201):65-158

Murray, J. 1991. Ecology and Palaeoecology of Benthic foraminifera. Longman Scientific Technical.UK. 397 pp. 
Murray, J. 2006. Ecology and applications Benthic Formainifera Cambridge University press. 426 pp.

Sen Gupta, B.K. \& M.L. Machain-Castillo 1993. Benthic foraminifera in oxygen-poor habitats. Marine Micropaleontology, 20:183-201

Smart, C. 2002. Environmental applications od deep-sea benthic foraminífera. IN Haslett. S (Ed.) Quaternary Environmental Micropalentology. Arnold, Inglaterra. $340 \mathrm{pp}$.

Tatian, M., J.C. Antacli \& R. Sahade 2005. Ascidians (Tunicata, Ascidiacea): species distribution along the Scotia Arc. Scientia Marina, 69 (2):205-214

Thompson, L. 1978. Distribution of living benthic foraminifera, Isla de los Estados, Tierra del Fuego, Argentina. Journal of Foraminiferal Research, 8 (3):241-257
Violanti, D., B. Loi \& R. Melis 2000. Distribution of Recent Foraminifera from the Strait of Magellan. First quantitative data. Bolletino Museo Regional Scientia. Naturalli. Torino, 17 (2):511- 539

Zapata, J. \& R. Alarcón 1988. Foraminíferos bentónicos del Estrecho de Magallanes (52 ${ }^{\circ}$ ' S; 69 54' W), Chile. Biota, 4:17- 29

Zapata, J. \& H. Moyano 1997. Foraminíferos bentónicos recientes de Chile austral. Boletín Sociedad Biología de Concepción, 68:27-37

Zelaya, DG (2005). Systematics and biogeography of marine gastropods molluscs from South Georgia. Spixiana, 28(2):109-139

Zúñiga-Rival, M. 2006. Estudio preliminar de los foraminíferos bentónicos (Protozoa: Foraminiferida) de bahía Yendegaia, Tierra del Fuego, Chile. Anales Instituto Patagonia (Chile), 34:33-40 